\title{
Correction to: the Inhibitory Effect of Chlorogenic Acid on Lipid Oxidation of Grass Carp (Ctenopharyngodon idellus) during Chilled Storage
}

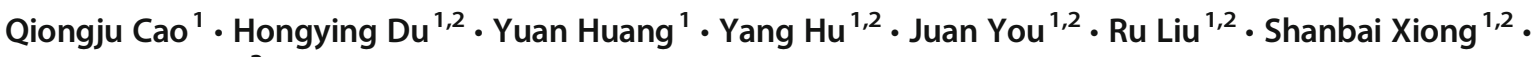 \\ Anne Manyande ${ }^{3}$
}

Published online: 2 January 2020

(C) Springer Science+Business Media, LLC, part of Springer Nature 2020

Correction to: Food and Bioprocess Technology (2019) 12:2050-2061

https://doi.org/10.1007/s11947-019-02365-0

The original version of this article unfortunately contained some mistakes.

The authors found that in the manuscript, the legends of Figs. 1, 3, 4 and 5 were not consistent with the figures, and the correct legends were showed as the following:

Fig. 1 Changes in conjugated diene value of grass carp treated with chlorogenic acid during chilled storage

Fig. 3 Changes in TBARS value of grass carp treated with chlorogenic acid during chilled storage

Fig. 4 Changes in FFA content of grass carp treated with chlorogenic acid during chilled storage

Fig. 5 Changes in COV of grass carp treated with chlorogenic acid during chilled storage

The online version of the original article can be found at https://doi.org/ 10.1007/s11947-019-02365-0

Hongying Du

hydu@mail.hzau.edu.cn

1 College of Food Science and Technology, Huazhong Agricultural University, Wuhan 430070, Hubei, People's Republic of China

2 National R \& D Branch Center for Conventional Freshwater Fish Processing, Wuhan 430070, Hubei, People's Republic of China

3 School of Human and Social Sciences, University of West London, Middlesex TW89GA, UK 